\title{
1 Idea paper: An envelope model of 2 ecological disturbance
}

3

4 Nicholas R. Friedman ${ }^{1,2}$

5

6 1. Environmental Informatics Section, Okinawa Institute of Science and Technology

7 Graduate University

8 2. To whom correspondence should be addressed

9

10

11 Keywords: disturbance, temporal dynamics, amplitude envelope, stability 


\section{Abstract}

14 Disturbance is common in natural ecosystems, but increasingly defines them. While there are many descriptions for the dynamics of an ecosystem's response to disturbance, there are few

16 descriptions for the dynamics of the disturbance itself. I describe a novel application of a

17 model based on the production of amplitude envelopes in acoustics and electronic music

18 synthesis, with varying parameters Attack, Decay, Sustain, and Release (ADSR). I show that

19 varying the parameters of the ADSR model is sufficient to produce and vary the qualitative

20 disturbance regimes described by previous authors, and is capable of producing dynamics not

21 previously considered. I tested the utility of the ADSR model by applying it to a logistic

22 growth model. I found that manipulating the attack and release parameters of the ADSR

23 model changes the population dynamics estimated by these models. This implies that

24 responses to disturbance are determined not only by the resilience and resistance of the

25 ecological system, but also the dynamics of the disturbance itself. My hope is that the ADSR

26 model will prove useful to researchers in either describing disturbances in long-term

27 ecological data, or in producing disturbances for simulations or experiments.

\section{Research Question}

30 How does variation in the temporal dynamics of ecological disturbance impact the characteristics of ecological response? 
34 Humans are altering the natural environment in many ways that perturb the planet's ecosystems (Vitousek, 1994) or disrupt natural disturbance regimes (Turner, 2010).

Ecological responses to disturbance vary widely, and researchers can use measurements such as resilience and resistance to characterize and in principle predict these responses (Donohue et al., 2013). Disturbance also varies widely, and its temporal dynamics can impact the trajectory of ecological responses (Jacquet \& Altermatt, 2020). A flexible model for generating or describing disturbance dynamics would contribute to our ability to test and predict the effects of various disturbances on ecological systems.

\section{Relevant Hypothesis}

In his seminal paper, Lake (2000; see also Bender et al., 1984) explored the temporal dynamics of disturbance in floods and droughts, and described three dynamics common in nature: "Pulse" events like floods, which are discrete and short-term, "Press" events like sedimentation, which are maintained at a constant level, and "Ramp" events like droughts, which steadily become more severe. While these categories have helped to shape ecologists' questions about disturbance (Ryo et al., 2019), many disturbances do not fit these shapes. Indeed, the temporal dynamics of flow rate during floods (Federer et al., 1990), radioactive iodine levels following the great Tohoku earthquake and tsunami (Inui et al., 2012), and atmospheric aerosols following volcanic eruption (Sato et al., 1993) all show a different disturbance pattern. This pattern is intense at first, rapidly receding, but returning to baseline only after several months or years. Thus, ecological research would benefit from a model of disturbance that is inclusive of these events, and in which temporal dynamics could be varied quantitatively in simulation or experimentation (see Ryo et al., 2019). While I did not 
succeed in finding such a model in the literature, my work in bioacoustics led me to one from sound and music production: the amplitude envelope (Beeman 1998).

\section{New Research Idea}

I describe a model for generating and discussing the temporal dimensions of ecological disturbance based on a popular component of electronic musical instruments - the envelope generator (Deutsch \& Deutsch, 1979). This component determines the change over time in its output (typically used to control amplitude), and generally uses four parameters to describe the shape of that change. These parameters, Attack, Decay, Sustain, and Release, are described in detail in Figure 1a-b. I will refer to them here as the "ADSR model". In music, this process is usually triggered by the press of a piano key. However, in its ecological application, I define the ADSR model to be a function of constant parameters describing the intensity of disturbance over time given a single input, the duration of a disturbance event. When the event beings, the function increases in intensity through the duration of the Attack stage to its maximum, and decreases through the duration of the Decay stage. The intensity then is held at the level of Sustain until the event is concluded, at which point the Release stage is triggered and continues for its specified duration. Three discrete states are implicitly assumed in the ADSR model: the original state, the maximum amplitude of the signal, and the sustained amplitude of the signal. However, the sustain state can be omitted by setting its value to 0 or 1 , and return to the original state can be omitted by continuing the event indefinitely. While there are assumed to be four temporal stages in the model, it can be truncated to only include Attack and Decay by setting the Sustain value to 0, and either the Attack, Decay, or Release stage can be omitted by setting their value to 0 . 
80 When applied to a simulation of ecological disturbance rather than sound amplitude, the

81 ADSR model is able to describe and generate the features associated with each of type of disturbance described by Lake (2000; Figure 1c). Using the model, one can reproduce "Pulse" disturbances using a short attack and release, and reproduce "Ramp" and "Press" disturbances using continued disturbance with varying attack. In addition to generating common categories of disturbance, the ADSR model should allow researchers to isolate and vary each parameter individually. Based on the dynamics described in many natural systems above, wherein an intense disturbance is followed by a slow return to the original state, I propose a new type of dynamic similar to the "Pluck" of a stringed instrument. This dynamic is intended to imitate the pattern of natural events described above: a brief and intense disturbance, followed by a prolonged recovery to the original state.

The terminology of the ADSR model may only be familiar to some musicians. However, unlike terms with more intuitive but potentially misleading names (e.g., stability and resilience; Donohue et al., 2016), the parameters of the ADSR model have meanings that are strictly quantitative, and so they should hopefully be clearer and more specific for use in discussion among ecologists.

99 Using the R programming environment, I built a simple script for generating ADSR models

100 for use in ecological simulation and modeling (script and demonstration are included in the

101 online supplement). In this implementation, I have assumed that change in intensity is linear

102 for the sake of simplicity. Researchers may wish to use exponential curves for their Attack, 
feature. Analog envelope generators are constructed of simple circuits, the length of each

107 which is described here in the supplement.

109 To test the effects of various perturbation dynamics on populations, I adapted Jacquet et al.'s

110 (2020) implementation of a logistic growth model with perturbation. This model assumes an

111 intrinsic growth rate that declines as the population approaches a carrying capacity. During

112 each time interval, the population grows - and in Jacquet et al.'s (2020) case a proportion of

113 the population can also be removed. While Jacquet et al. (2020) use this approach to

114 understand the effects of repeated perturbations, I re-configured their model to follow a

115 single perturbation whose intensity (fraction of the population removed) varies over time. To

116 evaluate the utility of the ADSR model, I estimated temporal dynamics of populations

117 responding to the disturbance dynamics described in Figure 1. These show different

118 outcomes depending on the parameters specified: "Press" and "Ramp" disturbances led to

119 near-immediate and eventual extinction, respectively, whereas "Pulse" and "Pluck"

120 disturbances led to immediate and eventual recoveries, respectively (Figure 2).

122 Furthermore, I used the ADSR model to estimate the dynamics of populations with similar

123 growth parameters and overall disturbance, but varying attack and release parameters. I found

124 that increasing attack reduced the impact on the population, and increasing release lessened

125 but lagged the impact (Figure 3a).

127 Lastly, I give a conceptual example of fitting an ADSR model to empirical ecological data

128 (Figure 3b), in this case the impact of spruce budworms on Canadian forests (Walker \& 
129 Taylor, 2020). Using the ADSR model, I describe this disturbance as having an attack and

130 decay stage of eight years each, with a release stage of twenty years - a dynamic that is a

131 poor fit to any previously described disturbance dynamic. Improvements in fit to these data

132 may be achieved by utilizing a long exponential decay stage in place of the release stage.

133 While the model in the figure is fit arbitrarily, it should be feasible to evaluate and optimize

134 the model's fit analytically (e.g., Caetano et al., 2010) or through a Bayesian-stochastic

135 approach.

137 One of the unique implications of "ramp" disturbance with a long Attack phase is the

138 potential for species to adapt to their newly altered environment, potentially buffering to

139 some extent the impacts of global climate change (Visser, 2008). To test the effects of

140 various disturbance dynamics on adaptation, researchers may employ evolutionary models

141 such as the multi-locus model developed by Jain and Stephan (2017). A preliminary test of

142 this model, as implemented by Johnsson (2021), showed apparent non-linear behavior in

143 response to varying values of release (see online supplement).

145 The results presented here ignore many important and relevant factors in ecological and

146 evolutionary dynamics, and are meant primarily to demonstrate the utility of the ADSR

147 model as a tool for future studies. Furthermore, researchers should exercise care in specifying

148 appropriate measurements of disturbance, and especially in separating disturbance from its

149 ecological response. My results are a hint of evidence that varying the dynamics of

150 disturbance, either qualitatively or quantitatively, changes its impact on populations.

151 Experimental studies, and especially empirical studies of long-term ecological data, are

152 needed that examine the resilience of populations and communities to disturbances of varying

153 temporal and also spatial characteristics (Ross et al., 2021). 


\section{Motivation}

156 Much of the impetus for this study comes from personal observations of ecological responses

157 to typhoons on Okinawa Island, Japan. Intense typhoons are brief even on an ecological time

158 scale, but they leave lasting effects that do not return to their original state for years (White et

159 al., 2017). Many animals may perish during the typhoon itself, but more still will find the

160 habitat of their home range no longer suitable, the forest canopy salted over and blown out

161 (Elliott \& Nino, 1960) or the coral reef damaged (Nanami \& Nishihira, 2002; White et al.,

162 2013). This motivated me to find a model of disturbance that included an intense event with a

163 slow subsequent recovery - a "Pluck" dynamic.

\section{Acknowledgements}

165 This paper is the result of conversations and thoughts had at the symposium on Ecological

166 Stability at the $66^{\text {th }}$ Annual ESJ meeting in Kobe, and especially with its organizers Sam RP-J

167 Ross and Yuka Suzuki. Ideas in this paper were improved by comments from Claire Jacquet,

168 Martin Johnsson, and Sam RP-J Ross, as well as conversations with members of the

169 Economo Unit, two anonymous reviewers and the editor. The author is supported by subsidy

170 funding to OIST.

173 The author declares no conflict of interest. 


\section{References}

175 Beeman, K. (1998). Digital Signal Analysis, Editing, and Synthesis. In Hopp, S. L., Owren,

176 M. J., Evans, C. S. (Eds.), Animal Acoustic Communication, (pp. 59-104). Heidelberg:

177 Springer-Verlag. https://doi.org/10.1007/978-3-642-76220-8_3

178 Bender, E. A., Case, T. J., Gilpin, M. E. (1984). Perturbation experiments in community

179 ecology: theory and practice. Ecology, 65, 1-13. https://doi.org/10.2307/1939452

180 Caetano, M., Burred, J. J., Rodet, X. (2010). Automatic segmentation of the temporal

181 evolution of isolated acoustic musical instrument sounds using spectro-temporal cues.

182 Proceedings of the 13th International Conference on Digital Audio Effects DAFx-10.

183 Deutsch, R., Deutsch, L. J. (1979). ADSR envelope generator. Journal of the Acoustical

184 Society of America, 66, 936. https://doi.org/https://doi.org/10.1121/1.383279

185 Donohue, I., Petchey, O. L., Montoya, J. M., ... Emerson, M. C. (2013). On the

186 dimensionality of ecological stability. Ecology Letters, 16, 421-429.

187 https://doi.org/10.1111/ele.12086

188 Donohue, I., Hillebrand, H., Montoya, J. M., ... Yang, Q. (2016). Navigating the complexity

189 of ecological stability. Ecology Letters, 19, 1172-1185. https://doi.org/10.1111/ele.12648

190 Elliott, J. C., Nino, Y. (1960). Okinawa’s dry typhoons. American Midland Naturalist, 63,

191 211-222. https://doi.org/10.2307/2422941

192 Federer, C. A., Flynn, L. D., Martin, C. W., Hornbeck, J. W., Pierce, R. S. (1990). Thirty

193 years of hydrometeorologic data at the Hubbard Brook Experimental Forest, New Hampshire.

194 U.S. 
Inui, T., Yasutaka T., Endo K., Katsumi, T. (2012). Geo-environmental issues induced by the

198 Jacquet, C., Altermatt, F. (2020). The ghost of disturbance past: long-term effects of pulse

199 disturbances on community biomass and composition. Proceedings of the Royal Society B, 287, 20200678. https://doi.org/10.1098/rspb.2020.0678rspb20200678

201 Jacquet, C., Gounand, I., Altermatt, F. (2020). How pulse disturbances shape size-abundance

202 pyramids. Ecology Letters, 23, 1014-1023. https://doi.org/10.1111/ele.13508

203 Jain, K., Stephan, W. (2017). Modes of rapid polygenic adaptation. Molecular Biology and

204 Evolution, 34, 3169-3175. https://doi.org/10.1093/molbev/msx240

205 Johnsson, S. (2021). A model of polygenic adaptation in an infinite population. Available at

206 https://github.com/mrtnj. Accessed June 4, 2021.

207 Lake, P. S. (2000). Disturbance, patchiness, and diversity in streams. Journal of the North 208 American Benthological Society, 19, 573-592. https://doi.org/10.2307/1468118

209 Nanami, A., Nishihira, M. (2002). The structures and dynamics of fish communities in an

210 Okinawan coral reef: Effects of coral-based habitat structures at sites with rocky and sandy

211 sea bottoms. Environmental Biology of Fishes, 63, 353-372.

212 https://doi.org/10.1023/A:1014952932694

213 Ross, S. R.-P. J., Friedman, N. R., Yoshimura, M., ... Economo, E. P. (2021). Utility of

214 acoustic indices for ecological monitoring in complex sonic environments. Ecological

215 Indicators, 121, 107114. https://doi.org/10.1016/j.ecolind.2020.107114 
216 Ryo, M., Aguilar-Trigueros, C. A., Pinek, L., Muller, L. A. H, Rillig, R. C. (2019). Basic

217 principles of temporal dynamics. Trends in Ecology and Evolution, 34, 723-733.

218 https://doi.org/10.1016/j.tree.2019.03.007

219 Sato, M., Hansen, J. E., McCormick, P., Pollack, J. B. (1993). Stratospheric aerosol optical

220 depths, 1850-1990. Journal of Geophysical Research, 98, 987-994.

221

https://doi.org/10.1029/93JD02553

222 Turner, M. G. (2010). Disturbance and landscape dynamics in a changing world. Ecology, 91,

223 2833-2849. https://doi.org/10.1890/10-0097.1

224 Visser, M. E. (2008). Keeping up with a warming world; assessing the rate of adaptation to climate change. Proceedings of the Royal Society B, 275, 649-59.

226 https://doi.org/10.1098/rspb.2007.0997

227 Vitousek, P. M. (1994). Beyond global warming: ecology and global change. Ecology, 75, 228 1861-1876. https://doi.org/10.2307/1941591

229 Walker, J., Taylor P. D. (2020). Evaluating the efficacy of ebird data for modeling historical

230 population trajectories of North American birds and for monitoring populations of boreal and

231 arctic breeding species. Avian Conservation and Ecology 15:1-43.

232 https://doi.org/10.5751/ACE-01671-150210

233 White, K. N., Ohara, T., Fujii, T., ...Reimer, J. D. (2013). Typhoon damage on a shallow

234 mesophotic reef in Okinawa, Japan. PeerJ, 1, e151. https://doi.org/10.7717/peerj.151

235 White, K. N., Weinstein, D. K., Ohara, T., ... Reimer, J. D. (2017). Shifting communities

236 after typhoon damage on an upper mesophotic reef in Okinawa, Japan. PeerJ, 5, e3573. 
(a)

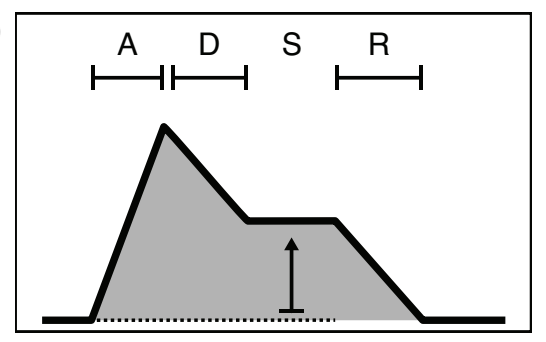

(b)

\begin{tabular}{|ll|}
\hline Attack & $\begin{array}{l}\text { Duration of the increase to the } \\
\text { maximum intensity }\end{array}$ \\
Decay & $\begin{array}{l}\text { Duration of the decrease to the } \\
\text { sustain intensity }\end{array}$ \\
Sustain & $\begin{array}{l}\text { Level sustained after A and D } \\
\text { while system is perturbed }\end{array}$ \\
Release & $\begin{array}{l}\text { Duration from end of perturba- } \\
\text { tion to original state }\end{array}$ \\
\hline
\end{tabular}

239

240 Figure 1: Explanation of the ADSR envelope model (a) with parameter definitions (b);

241 perturbation types described by Lake (2000), and one described in this paper, are reproduced

242 using the ADSR model (c), with parameter values included. Dotted lines indicate the duration

243 of perturbation.
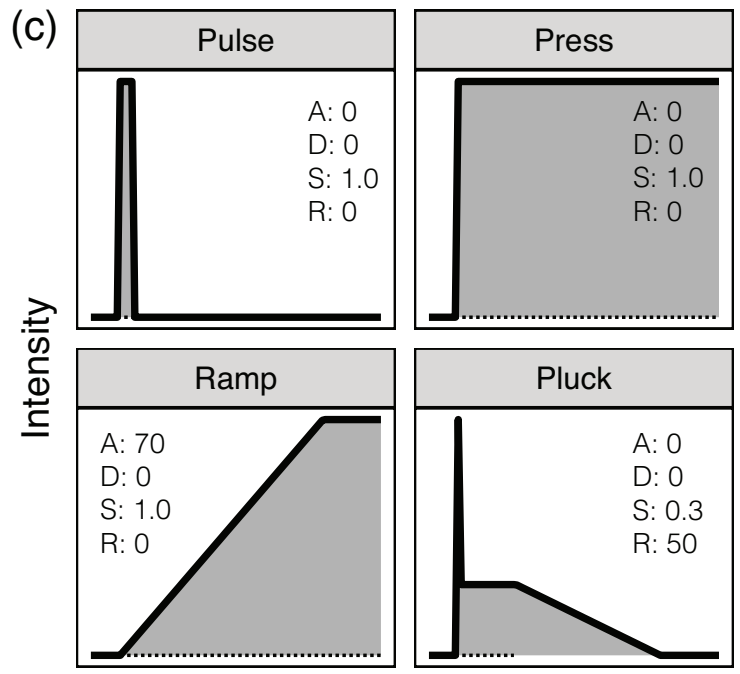

Time 


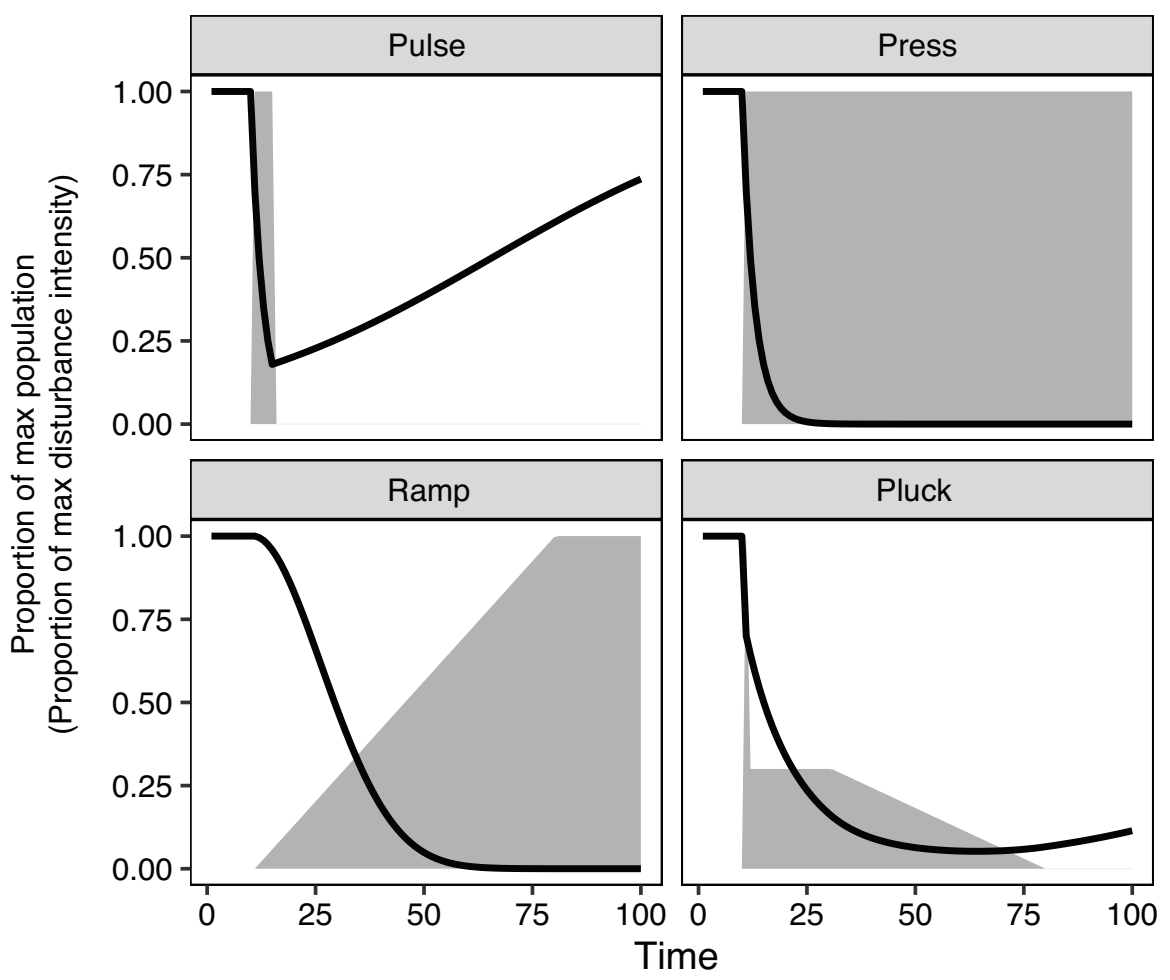

246 Figure 2: Population effects of disturbance dynamics with types described by Lake (2000),

247 and one described in this paper. The black line represents the proportion of the initial

248 population remaining, while the grey polygon represents the proportion of the maximum

249 disturbance intensity. Disturbance is simulated here by removing $30 \%$ of the population per

250 time increment at maximum intensity. 

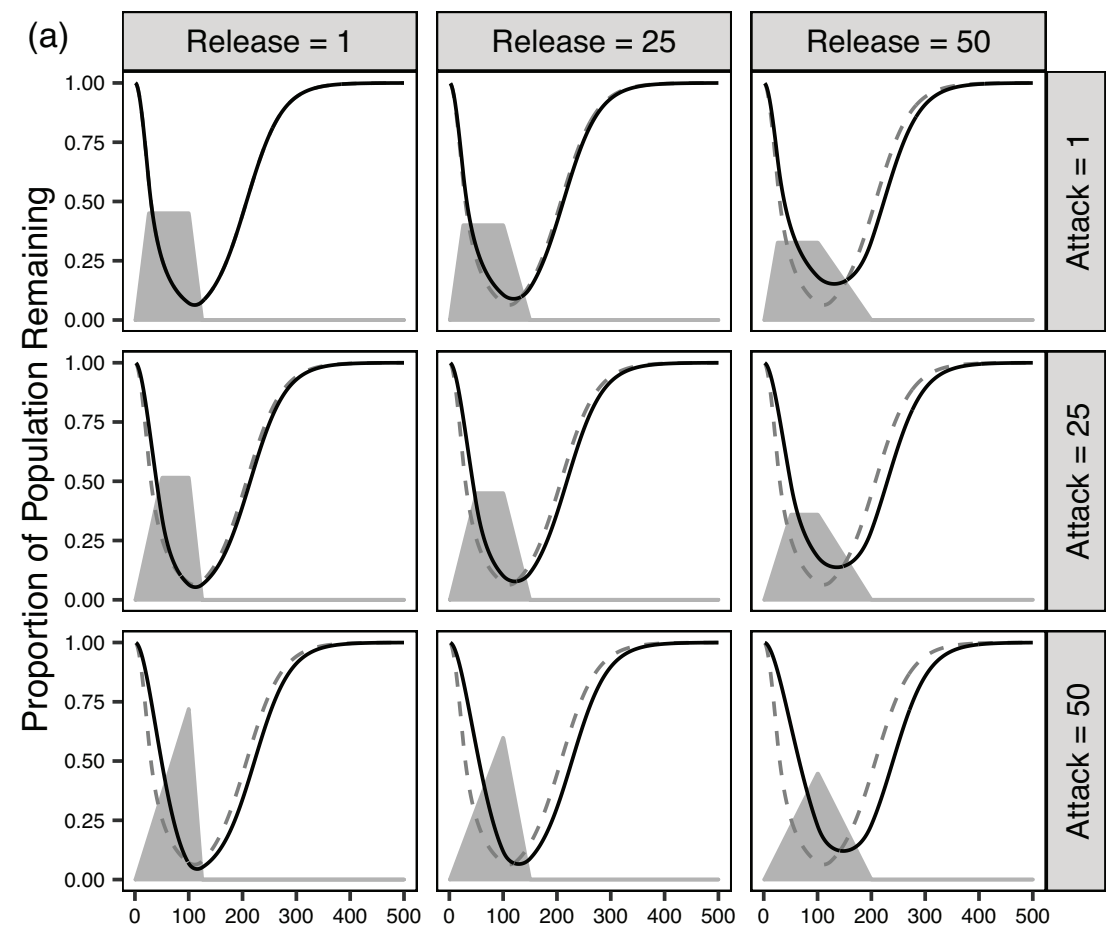

Time

(b)

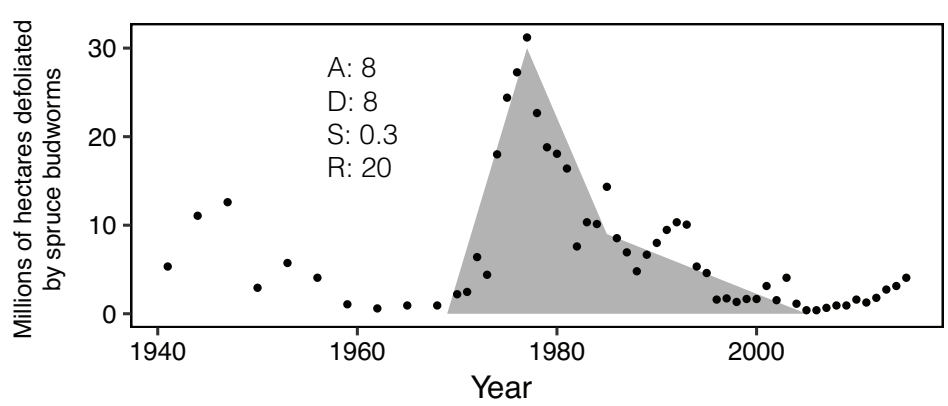

253 Figure 3: Population effects (a) of disturbance dynamics with varying attack and release

254 parameters (polygons shaded light grey) and their effects on population dynamics assuming a

255 logistic growth model (black line; Jacquet et al. 2020). Dynamics from the upper left panel

256 are included in each subsequent panel as reference (dotted line). Disturbance amplitude

values are adjusted to ensure that the area under the curve is approximately equal (models

without this adjustment also showed effects of varying attack and release parameters). An

example (b) of an ADSR model fit to empirical ecological data, in this case the intensity of a

260 spruce budworm outbreak in North America (re-plotted with permission from Walker \&

261 Taylor, 2020). The grey polygon outlines the model with its parameters given at left, and the 